\title{
La Ludoteca tiene aguante. Una experiencia de Trabajo en Dispositivo Lúdico Grupal con niños y niñas*
}

\section{The playroom can stand a lot. A work experience in a group playful system with children}

\author{
Denise Silbermann $^{\star \star}$
}

\section{Resumen:}

Este artículo analiza un dispositivo lúdico grupal con niños y niñas de 5 a 13 años, que se desarrolló entre los años 2012 y 2013 , en la Ciudad de Rosario. El análisis de esta práctica se realizó a partir de la población de niños y niñas que demandaron ingreso y atención en el dispositivo. La metodología elegida se enmarca en una estrategia mixta, a través de un Estudio de Casos. El objetivo fue dejar un registro escrito y una sistematización de la experiencia a fin de enriquecer las intervenciones y compartir el resultado con la comunidad, equipos locales, y otros interesados en la temática.

Palabras claves: niños - niñas - dispositivo grupal - juego - tiempo

\section{Abstract}

This article analyzes a group playful system with boys and girls aged from 5 to
13 years, which was developed between 2012 and 2013, in the City of Rosario. The analysis of this practice was carried out on the basis of the population of children who requested admission and care in the system. The methodology chosen is part of a mixed strategy, through a case - study. The objective was to prepare a written - record and systematize the experience in order to enrich the interventions and to share the results with the community, local teams and other stakeholders.

Keywords: Boys - Girls - Group System - Games - Time

\footnotetext{
* Artículo escrito sobre la base del Trabajo Integrador Final para la Carrera de Especialización en Psicología Clínica, Institucional y Comunitaria de la Universidad Nacional de Rosario. El TIF, dirigido por Lic. Miriam Giani, fue presentado en 2014 y aprobado en 2015.

** Psicóloga. Especialista en Psicología Clínica, Institucional y Comunitaria (UNR). Ex Docente de la Cátedra de Psicología Educativa II, Facultad de psicología, UNR. Miembro del Equipo Psicosocial del Hospital de Junín de los Andes, Neuquén. Docente de la Carrera de Especialización en Enfermería Universidad del Comahue. Coordinadora del dispositivo Grupal de mujeres en situación de violencia "Ronda de Mujeres". Directora de la Investigación "Aspectos psicológicos y socio-culturales del fenómeno del suicidio en Junín de los Andes 20162018”.denisesilbermann@gmail.com
} 
muchas experiencias en dispositivos grupales, son escasos los registros y poca la producción teórica y clínica al respecto. Este Espacio de Juego, se sostuvo durante varios años, con una población estable y sistemática de niños y niñas.

A modo de resguardo de la confidencialidad y del anonimato, y en coherencia con la ética y la lógica propia del trabajo realizado en aquel dispositivo grupal, en este artículo tanto las referencias institucionales, como los nombres de las niñas y los niños, han sido preservados. Entendiendo que estas abstenciones y resguardos, son también un modo de cuidado al otro.

La propuesta de llevar adelante este Espacio de Juego Interistitucional surgió como proyecto de los trabajadores de un Centro de Salud y un Centro de Comunitario Estatal) de la zona oeste de la ciudad de Rosario. Teniendo en cuenta las situaciones recibidas y trabajadas en ambas instituciones, los equipos de trabajo fuimos construyendo un diagnóstico acerca de la infancia en ese barrio en particular.

La mayoría de las consultas que habían llegado al servicio de salud mental y al Centro Comunitario referían a situaciones de niños y niñas que tenían como denominador común la situación de riesgo y vulnerabilidad. En su mayoría se encontraban desafiliados de las instituciones, habían dejado de asistir a la escuela o lo hacían de forma irregular, el único lugar que se les presentaba era la calle, la esquina.

\section{Listos, preparados...Ya}

El Espacio de Juego tuvo la intención de trabajar con aquellos niños y niñas que no habían podido tomar otros dispositivos de infancias, y también fue formulado como espacio que desplegara un abordaje preventivo. Se observó que aquellos niños $\mathrm{y}$ aquellas niñas que quedaban por fuera de algún tipo de alojamiento familiar, comunitario o institucional, eran los y las que llegan al espacio de juego a través de una consulta o por derivaciones. Niños y niñas que, desde muy pequeños, no participaban en forma directa, pero sí estaban inmersos en circuitos delictivos (bunkers) y prostitución. Complejizando aún más, nos encontramos con situaciones de "problemas de aprendizaje", "problemas en el lenguaje", "problemas de conducta", situaciones de violencia y abuso intrafamiliar, duelos por migraciones, fragmentaciones familiares $y$ fuertes inhibiciones.

En un punto opuesto, y como contexto de crianza, nos encontramos con niños y niñas que ante un exceso de cuidado no tenían permitido salir de sus casas (en algunos casos tampoco a la escuela), o en otros en donde el desvalimiento de las figuras parentales era tal, que podríamos poner una pregunta a si nos encontrábamos frente a niños y niñas o a pequeños adultos cuyas responsabilidades excedían a su edad cronológica y a sus posibilidades subjetivas.

Consideramos fundamental, entonces, propiciar un espacio en donde se dieran las condiciones para que algo del orden del juego pudiera comenzar a desplegarse.

Es ya en el marco de la carrera de especialización, en el espacio de Residencia, que surge una frase contundente: "las Ludotecas son aguantaderos de pibes". Esta frase comenzó a rodar muchas preguntas, interrogantes... ¿Qué espacio había en la 
carrera para compartir las vicisitudes de éstas, nuestras prácticas? ¿Qué territorios se estaban entramando que requerían de nuevos dispositivos de abordaje? ¿A qué era a lo que se le hacía aguante en las ludotecas? ¿A qué se le hacía borde en ese dispositivo grupal de niños y niñas? Necesitábamos repensarnos como profesionales, lograr un detenimiento para contestar esa frase, y relanzar nuevas intervenciones. La escritura, la sistematización en un TIF, posibilitaría re-inscribir, re-visar, re-leer la particularidad de este dispositivo de juego en territorio.

\section{Un paseo por el espacio de Juego}

Hacia el año 2012, propusimos un espacio grupal de juego para niños/as de entre 5 y 13 años. Con un cupo de 10 y un máximo de 15 integrantes. En un primer momento, coordinábamos el espacio dos psicólogas y un médico residente. Más tarde, se sumó, una residente de la Carrera de Posgrado en Psicología Clínica Institucional y Comuni$\operatorname{taria}^{2}$.

Dicho espacio funcionaba en el SUM del Centro de Salud, los días miércoles de 13:30 a 15 hs. Cuando pensamos en este proyecto intentamos contestar las siguientes preguntas: ¿Por qué un espacio lúdico en un centro de salud? ¿Por qué una articulación entre secretarías? Algunos de los intentos de respuesta fueron generar propuestas dentro del centro de salud que no tuvieran que ver

\footnotetext{
${ }^{1}$ En la cohorte posterior, la carrera incorporó un seminario específico sobre prácticas y dispositivos grupales.

${ }_{2} \mathrm{Al}$ momento de terminar este trabajo, se había sumado al espacio una residente de pre-grado de la cátedra de Residencia Cínica de la carrera de Psicología UNR,
}

con la enfermedad; la construcción de otra noción de la salud, referida a la vida, al juego, a la cultura.

Otras de las respuestas apuntaron a afianzar los lazos con la comunidad. De hecho, muchas intervenciones y acercamientos a las familias se pudieron dar con mejor aceptación y escucha cuando venían a través del espacio de juego. En la misma línea, se trabajó para permeabilizar la institución, interrogando nuestros modos de abordaje, procesos de trabajo y elaboración de estrategias clínicas; propiciar un espacio interinstitucional desde donde acotar los tiempos y agilizar las intervenciones en los casos que así lo requieran.

Es por lo expresado hasta aquí que, para este equipo, fue fundamental ofrecer un espacio de juego que propiciara el desarrollo de las posibilidades de simbolización, socialización y tramitación para los niños y niñas del barrio, teniendo como propósitos, brindar un espacio grupal en articulación a un seguimiento de las singularidades de los niños y niñas; crear instancias de trabajo interdisciplinario que generan una otra escena respecto de la atención en el consultorio; propiciar abordajes integrales de cada caso singular donde pudiésemos construir una mirada desde las distintas disciplinas para no caer en cristalizaciones disciplinarias; funcionar como una instancia desde donde construir un diagnóstico de situación en ambos planos, singular y colectivo acerca de la infancia en el barrio.

El Espacio de Juego fue un dispositivo lúdico grupal infantil, no una mera inclusión en un taller. Se establecieron criterios de ingreso, un encuadre de trabajo y una direccionalidad en las intervenciones: en 
lo Singular, en lo Grupal, en lo Familiar y en lo Comunitario. Todas estas dimensiones serían fundamentales para entender $y$ llevar adelante esta práctica. A lo largo de esos años, desde la apertura en el año 2012, aquel fue un espacio que fue ganando reconocimiento, tanto a nivel de las familias de los usuarios y vecinos, de los profesionales de las instituciones, como lugar de valoración, que recuperaríamos en las entrevistas y reuniones de padres.

Durante todo ese período se llevaron a cabo distintos modos de registro de lo sucedido, tanto a la hora de llevar adelante el dispositivo, como todo el trabajo en torno al mismo. Si bien contábamos con información muy valiosa, a la hora de intentar alguna transmisión, justamente debido a la riqueza del proceso y al tiempo de trabajo que ya llevaba varios años, no podíamos lograrlo. Por ello, este primer intento de sistematización de esta información permitió revisar las prácticas a fin de mejorar la atención y las intervenciones, pudiendo producir un detenimiento en la vorágine de las actividades diarias.

Con esta producción se buscó transmitir a compañeros de las instituciones involucradas (pediatras, educadores, trabajadoras sociales) el trabajo realizado, ya que de lo contrario muchas veces solo quedaban en relatos aislados, en registro en historias clínicas o anécdotas. La sistematización y la transmisión de la información de forma ordenada, ayudaron a que todos los trabajadores pudieran conocer mejor el dispositivo y tomarlo como una herramienta más a la hora de contar con un espacio o estrategia para el trabajo con algún usuario y su familia. Además, se consideramos que el aporte de esta información enriquecía las evaluaciones de los procesos de trabajo de los equipos de las dos instituciones involucradas en esta propuesta.

Sabíamos que había habido espacios similares (relatos compartidos por compañeros de la carrera de grado y posgrado, experiencias en otros barrios y otras instituciones) algunos sin continuidad o de los que no se había podido dejar constancia de esos trabajos.

Uno de los ejes fundamentales que tomamos a la hora del armado y ejecución de este dispositivo fue la participación en el proceso, incluidos los momentos de evaluación y pasaje a otras propuestas (como la colonia en verano), de los padres y familias de los niños y niñas que asistían.

Algo que insistió, retornando tanto en los decires de estas familias como en los niños, captó nuestro interés y se tornaron en preguntas:

T.G. niña de 6 años: “¿Cuándo era mi cumpleaños?”.

U.B. niño de 6 años: “¿El próximo miércoles...mañana?".

G.T niño de 7 años: "Mañana, ¿cuándo estábamos jugando a ese juego te digo?". Haciendo referencia al miércoles anterior.

T.G. niña de 6 años, iba todos los días preguntando si era miércoles. (Día del espacio de Juego).

M.M. niño de 10 años: "Yo sabía que era hoy, pero me acordé tarde...no me puedo acordar antes de la hora...¿Me avisás?”

Mamá de M.M. niño de 10 años: “Me podrás hacer acordar cuándo 
sea el día y la hora, estoy perdida". Mamá de L.P de 13 años: "Antes no quería venir, estaba siempre tirado, ahora pregunta si es miércoles y qué hora es".

¿A qué se corresponderían entonces estos decires: confusiones, momentos de construcción, lapsus, diferencias culturales? ¿Qué aparece en esas coordenadas de un tiempo acordado y respetado, en un contexto donde estalla la discontinuidad y no se pudieron poner palabras?

En un contexto de vulnerabilidad en donde es el "ahora", la inmediatez lo que permite la subsistencia, ¿cómo se irían instalando, entonces, las categorías de pasado y futuro?, ¿qué ocurre con estos niños en donde un real en el cuerpo biológico viene dejando marcas, silencios, marcando tiempos? Por ejemplo, "Salió el turno para la resonancia, es el viernes". Un niño pudo decir en el espacio grupal que estaba asustado, porque nadie le había explicado qué le iban a hacer, tampoco quién iba a estar allí.

Otra pregunta insiste. ¿Cómo un encuadre, reglas establecidas por el grupo, confidencialidad y el cumplir con esto, va recortando un espacio en donde a través del jugar se pueda ir desplegando la conformación de un tiempo? Ese tiempo va entramando otras posibilidades. Algo de la presencia sostenida e interesada ¿empezará a dibujar algún recorte en relación a lo temporo-espacial?

\section{Algunas coordenadas teóricas}

Consideramos imprescindible hacer un recorrido de algunos conceptos en lo refe- rente al jugar, en un espacio de juego grupal y en tensión con el tiempo como categoría, dado que son fundamentales para enmarcar este análisis.

En principio, es preciso hacer una distinción entre juego y jugar, distinción que ya marca Ricardo Rodulfo (1999), en tanto:

Insisto en la importancia de decir Jugar y no juego, siguiendo la propuesta de Winnicott, para acentuar su carácter de practica significante que tiene para nosotros esta función, en tanto el juego remite al producto de cierta actividad, a un producto de determinados contenidos, la actividad en si debe ser marcada por el verbo en infinitivo, que indica su carácter de producción. (p. 120)

En otro pasaje, Rodulfo (1999) plantea al juego como hilo conductor para no perderse en la compleja problemática de la constitución subjetiva:

Partimos de un descubrimiento: no hay ninguna actividad significativa en el desarrollo de la simbolización del niño que no pase vertebralmente por aquel. No es una catarsis entre otras, no es una actividad más, no es un divertimento, ni se limita a una descarga fantasmática compensatoria o a una actividad regulada por las defensas, así como tampoco se lo puede reducir a una formación del inconsciente: más allá de estas parcialidades, no hay nada significativo en la estructuración de un niño que no pase por allí, de modo que es el 
mejor hilo para no perderse. Los conceptos más abstractos o genéricos (como el deseo y tantos otros) que podamos invocar, bienvenidos sean, pero ¿dónde voy a verlos funcionar si es que funcionan, dónde comprobaré su pertinencia si no es en esta práctica por excelencia? En particular, cada vez que quiero evaluar el estado de desarrollo simbólico de un chico, no hay ningún índice que lo brinde más claramente que el estado de sus posibilidades en cuanto al jugar". (p. 121)

Sabemos que es fundamental la construcción de rutinas para todos los niños y niñas.

Las rutinas son otros nombres de la fabricación de superficies: cabe el otro primordial ofrecer por medio de ellas los medios para armar una cotidianeidad (...) Nada más y nada menos que la rutina, es decir algún tipo de banda, una superficie de rutina, armada la cual puede pasarse a otra cosa (...) Lo que la vieja psicología estudiaba bajo el nombre de hábitos, para nosotros los psicoanalistas, es parte de una función mucho más trascendente porque constituye una retícula de soportes narcisistas en lo que toda subjetividad necesita apuntalarse (...) La moraleja es, que cuando no ha quedado superficie organizada, hay que construirla (restitutivamente) día por día con lo que se pueda. (Rodulfo, 1999, p.136)
Podríamos decir, entonces, que la continuidad y el marco dado al espacio de juego grupal fueron condiciones fundamentales para su constitución y para que lo allí acontecido tenga efectos. Al ofrecer este recorte de un tiempo que a la vez es continuo y permitió cierta previsibilidad, pero que al mismo tiempo dejó abierta la brecha de lo espontáneo-, es que algo del orden del juego se pudo desplegar.

Alfredo Moffatt (1991) propone el concepto de psiquismo acrónico-conciencia sin temporalidad-como concepto básico para entender la cosmovisión de un ser que crece sin figuras paternas y sin un hábitat propio integrador del proceso de vida:

La identidad precaria que puede construir está basada sólo en un estilo de acción, no en una historia; dentro de una ranchada juega como una pieza dentro del grupo de la sobrevivencia y adquiere un alias que es su nombre: Chapita, Pelado, Huesito... En general no tienen documentos, la figura de la madre es un recuerdo lejano y pocos conocieron al padre. Si analizamos las características de personalidad que tienen, están definidas por: 1) la no historicidad - no memoria y no proyecto de vida- lo que llamamos psiquismo acrónico (cronos=tiempo), 2) lenguaje de acción, no simboliza sino que hace, actúa como comunicación, 3) la sobrevivencia está basada en la mendicidad o pequeños robos (no tiene otra salida) y 4) consiguen alivio por drogas (especialmente pegamentos). Si analizamos estas cuatro estrategias 
de sobrevivencia, vemos que para las condiciones de abandono extremo son cuatro soluciones correctas, terribles pero inevitables: acronicidad, para evitar la angustia de la muerte, porque no hay historia por lo tanto no hay conciencia de finitud; lenguaje de acción, no hubo aprendizaje de la simbolización; violencia transgresora, el débil no sobrevive en la calle y se droga para no psicotizarse. (p.1)

Pero entonces, ¿cuáles serían las líneas para poder pensar un trabajo posible? Es desde el mismo concepto de acronicidad psíquica que Moffatt (1991) propone pensarlo:

El psiquismo existe como autopercepción de identidad si el yo se ve reflejado en la mirada del otro (del tú) dentro de un vínculo. Esto debe suceder en un campo de dos dimensiones: espacio y tiempo, es decir dentro de un hábitat e intercalado en una secuencia temporal. Dicho de otra manera, en un adentro, un recinto que recorta la escena y en un presente, que generando un corte en el devenir de sucesos defina un antes y un después - pasado y futuro-. La memoria se extiende hacia delante, se organiza una expectativa, un futuro, un proyecto. (p.2)

Por último, retomamos el mientras tanto, conceptualización que gustaba aplicar Fernando Ulloa, y que da cuenta de una dimensión posible del trabajo.
En la concatenación de escenas, multiplicación dramática, van ensayando distintas salidas posibles, sociabilizando lo traumático.

Lo terapéutico se centra en que, aún en las peores condiciones, cuando un niño puede jugar, sostenido desde un adulto, existe la posibilidad de pensar una manera distinta de vivir, con ello no estamos diciendo acá no pasa nada, nuestra premisa es trabajar de la desesperanza a lo posible, en el "mientras tanto. (Basile, 2012, p.206)

Para que un niño pueda jugar tiene que establecerse un espacio confiable (Basile, 2012), pues entonces aparece la pregunta ¿cómo poder garantizar esto en contextos de inestabilidad y desamparo?

En una carta, Fernando Ulloa dirá:

Me reitero diciendo que esta sugerencia aspira a lograr que el equipo de Barriletes implemente, pertinentemente, la transformación de ese como si en un mientras tanto necesario y explícito, a partir del propio esfuerzo por construir un devenir. El devenir, como abarcativo de temporalidad humana, construye desde la presencia de un presente como el de Barriletes, que permite resignificar pasado e ir bosquejando mañanas.

Esta fuerte presencia de un presente es un antídoto contra un pasado que se actualiza continuamente, malogrando futuro.

Este presente es un acontecimiento que marca un antes y un después, un 
corte desde el cual se puede superar el carácter traumático de ese pasado y bosquejar un futuro. (Ulloa, 2006 en Basile, 2012, p.101)

\section{Trazos, marcas, registros y escrituras}

Recuperamos, para la realización del trabajo, los soportes de registros con los que veníamos trabajando en el espacio lúdico:

- Registros del dispositivo por encuentro: este registro se realizaba una vez terminado el encuentro, turnándonos los coordinadores para la escritura del mismo, participando todos de la realización, ya que por momentos se daban dentro de la misma escena grupal, sub-escenas, de las que no todos estamos al tanto, y compartimos lo sucedido y cómo se intervino y que ocurrió con los que participaban de las misma. Se registraban las situaciones de juego, decires de los niños, intervenciones.

- Planilla individual de cada niño: en dicha planilla se ingresaban y actualizaban datos como domicilio, escolaridad, constitución familiar, estado de salud, medicaciones si las recibe, y observaciones que nos parecían pertinentes.

- Historias clínicas: son las carpetas del grupo familiar con las que cuenta el personal del Centro de Salud. Para nosotros, constituyeron un registro más de consulta. Allí sólo hacíamos registros puntuales luego de discutirlo en reunión, ya que esas historias clínicas son de acceso público. Se registra el momento de ingreso al espacio, la propuesta inicial de trabajo, y el seguimiento ( $\mathrm{si}$ asiste, si se lo o la reconvoca, si no quiere participar, si alguno de los padres resiste la propuesta).

- Planillas de asistencias mensuales: en donde constaba la fecha del encuentro y los niños asistentes, también si el encuentro se suspendió y por qué motivo (paro, feriado, toma del centro de salud, otros).

- Registros de las entrevistas individuales con los padres, madres o referentes: las entrevistas eran programadas según el trabajo que se venía dando con cada participante del espacio y su familia. La participación de los coordinadores era formulada de acuerdo a la estrategia, que se discutía en relación a las transferencias e implicancias puestas en juego con cada uno de los miembros del equipo.

- Registros de las reuniones con los padres, madres, referentes: uno de los coordinadores tomaba registro $y$, por tanto, quedaba en un lugar de observador. Las otras dos personas coordinaban el encuentro en base a un temario ya preparado con anterioridad, así como también se abría un tiempo de evaluación conjunta y de escucha a las problemáticas que los familiares pudieran plantear. Se realizaban como mínimo tres reuniones al año, generalmente, una al inicio; una antes o después de las vacaciones de invierno $y$, hacia fin de año, otra en el pasaje a la colonia de verano. 
- Cuaderno de derivaciones: este es un modo que instalamos de agendar posibles derivaciones al espacio. Surge como un intento por interrumpir las derivaciones de pasillo o de cocina; que si bien entendíamos que eran espacios de encuentro dentro de las instituciones, no podíamos naturalizarlas como modalidad de trabajo. Cada profesional, cada vecino o usuario que había considerado al espacio de juego como un lugar posible de trabajo con un niño o niña, un tiempo de escucha. Se dejaba registrada, entonces, esa posible derivación, y luego se agendaba un momento de encuentro para desplegar la situación.

\section{La planilla de sistematización de datos}

La planilla para sistematizar los datos fue construida oportunamente para el relevamiento del trabajo, se puso en discusión por el equipo del espacio lúdico, entre quienes planteamos que debía contener datos que permitieran conocer a los adultos referentes del niño (si los había), origen de su familia, condiciones de escolaridad, ubicar los motivos por lo que se pensó cada ingreso y quién lo hizo, plasmar las primeras impresiones del equipo frente a la derivación, ubicar las estrategias de trabajo, conocer y cotejar la situación de ingreso, las intervenciones y el estado actual del niño a la hora de realizar el trabajo.

La planilla constó de seis partes: 1) Recolección de datos personales: edad, sexo, escolaridad. 2) Relevamiento de datos demográficos: personas a cargo del niño, lengua materna y hablada. 3) Registro de información respecto del espacio lúdico: fecha de ingreso, cantidad de encuentros presentes. 4) Relevamiento de las derivaciones, motivos de consulta. 5) Registro de la información sobre la modalidad misma de trabajo del espacio lúdico: Hipótesis diagnostica, material recuperado en torno a la categoría de tiempo, estrategias de trabajos y efectos si se pueden ubicar. 6) Pasajes y egresos: continuidad durante la colonia de verano.

\section{Algunos de los resultados a compartir}

Fuimos encontrando cambios sustanciales en torno a las demandas iniciales y los trabajos realizados junto a profesionales tanto del centro como de otros organismos e instituciones. Asimismo, quedaron plasmados los efectos del trabajo con las familias en torno a construir otros modos de acompañar estos procesos de subjetivación de estos niños.

De la población asistente, la distribución por sexo era equitativa, como así la edad, notándose apenas en número superior la franja de 5 a 7 años, por sobre la de 8 a 13 años. Sobre ese total de niños y niñas la mayoría asistía en forma regular a la escuela, sólo tres lo hacían en forma irregular y dos nunca habían asistido a ninguna institución educativa. Y en cuanto a la elección de las familias por las escuelas del barrio o fuera de él, arrojó que esta distribución era en partes iguales.

En cuanto a la constitución familiar, y en oposición a otro imaginario del equipo, ocho de las trece familias, están constituidas por ambos padres. En las otras cin- 
co aparecían como sostén de familias las madres, y de estas hay tres en donde los papás estaban detenidos. Las principales actividades productivas consignadas eran el cirujeo y las changas. Sólo dos padres tenían trabajos regulares. La mayoría de las familias contaban con la Asignación Universal por Hijo.

Si bien la lengua hablada en todas las familias era el castellano, dos de ellas, además del castellano, hablaban guaraní (dialectos diferentes: uno de Misiones, el otro, de Paraguay) y sólo una conservaba la lengua Q'om en su cotidianidad.

Otro dato importante, fue el de los ingresos al espacio. Durante el primer año se registraron nueve, en 2013 sólo fueron cuatro. Este dato cotejado con la planilla de sistematización, da cuenta de que la mayoría de los niños continuó durante el segundo año. Se realizaron sólo dos propuestas de un trabajo previo individual para trabajar cuestiones previas al ingreso a la escena grupal. Esos ingresos se concretaron para el momento de la realización del TIF. Sólo hubo un caso, de una niña, que no quiso participar del espacio y, por otro lado, se logró el pasaje de uno de los niños que había cumplido 14 años, a otra propuesta acorde a sus intereses propios de su edad.

Otro dato no menor, y en oposición a lo que esperábamos cuando iniciamos el dispositivo, es que los motivos de las consultas fueron, en su mayoría, problemáticas ligadas a inhibiciones y dificultades con los pares. Pero también se encontraron situaciones familiares complejas y, finalmente, situaciones traumáticas ligadas a cuestiones de salud. Sólo en dos casos se refirieron como motivo problemáticas ligadas a lo escolar.

A partir de este análisis de datos nos encontramos con la sorpresa de muchas derivaciones provenían desde el equipo del centro de salud (generalistas y pediatras). Caracterizamos como sorpresa este hallazgo ya que en el imaginario del equipo del espacio se suponía no contar con derivaciones internas del mismo. Estas derivaciones son una posibilidad de ampliar y enriquecer una estrategia de trabajo con ese niño o niña y esa familia, así como un recurso para realizar un diagnóstico interdisciplinario.

Es importante señalar que se pudieron ordenar las hipótesis diagnósticas de trabajo en dos grandes núcleos: Preguntas en torno al lugar de ese niño en la trama familiar, y Preguntas en torno a diagnósticos indefinidos, poco claros o en confirmación y sus consecuencias en estos niños en proceso de subjetivación.

En lo que respecta a otros de los objetivos propuestos en el trabajo, que consistió en ubicar cómo se iban desplegando las dimensiones de lo histórico- temporal en las escenas lúdicas y en los decires de los niños y niñas, no se encontraron mayores dificultades respecto a lo esperado en cada una de las etapas del desarrollo, salvo las que tenían que ver con la imposibilidad de ubicar el recorrido en Tiempos históricos, esta construcción estaría en proceso de consolidación hasta los 6 y 9 años. Y siendo la dificultad en diferenciar y utilizar los términos Ayer y mañana, la que apareció en 3 de los niños/as. Y si bien aún para la edad de 3 años, estos términos no son utilizados adecuadamente, los/as que presentaron esta dificultad y en forma insistente fueron niños mayores a 6 años. 


\section{Pido gancho...a modo de conclusión}

Este trabajo estuvo centrado en poder analizar el dispositivo lúdico grupal con niños y niñas, a través del recupero del material con el que ya se contaba en multiplicidad de soportes, se hizo hincapié, en ubicar los decires de los niños, sus despliegues de escenas lúdicas, como del aporte realizado por sus padres y familias en constante vinculación.

Se trató, entonces, de pensar y alojar las condiciones en la que crecían y vivían estos niños, y desde allí, significar el lugar que ocupó y ofreció este dispositivo. Estas rutinas garantizaron estabilidad y permanencia a niños y niñas sin palabra, sin historia viviendo el instante. Prestarle historias, decía Ulloa (2012), historia de un espacio, de los coordinadores, historias de paseos y de verano en la colonia, de los que pasaron y ya no estaban pero que dejaron relatos, dibujos, fotos, marcas, hacen posible que puedan seguir construyendo la propia.. "Yo venía acá cuando tenía 5 años". "Yo fui a ese paseo". "Yo tengo las fotos de los paseos". Surgieron palabras que ligaron, tiempos ligados-ligantes.

Remarcamos, también, estos intervalos en la cotidianeidad del trabajo, que implicó detenerse a escribir, estudiar, discutir, socializar y elaborar a partir de la práctica. Fueron, también, tiempos y espacios de producción de salud para los trabajadores.

Durante el tiempo de elaboración del TIF, en el barrio hubo momentos muy difíciles: saqueos, robos, la llegada de la Gendarmería como actor y personaje en las calles ${ }^{3}$. Estallaron bombas de tiempo, situaciones

\footnotetext{
${ }^{3}$ A partir de una situación de violencia entre un
}

que se venían planteando como preocupantes. De muchas instituciones quemadas, robadas y estalladas allá por el diciembre de 2013, dos lugares se preservaron por los vecinos y por los niños: uno fue el Centro de Salud y el otro, la Ludoteca.

No se llevaron las fotos, no se tocaron los afiches colgados, no se llevaron los juegos, las producciones de los chicos estaban intactas. ¿Violencias institucionales, que retornaron contra las instituciones? Ese día no queríamos abrir la puerta de la sala. Pensábamos que estaba como las otras instituciones, destrozada, sucia. Nos tomamos unos minutos, y al entrar, p. Parecíamos haber cruzado otra dimensión: todo estaba en su lugar. Las emociones emergían., Algunos compañeros hacían el chiste de: " ¿Che, hicieron algún arreglo ustedes, que no les tocaron nada?" Al parecer sí, algo hizo de ese lugar otra cosa, diferente al menos a la serie de las otras instituciones. Aquel espacio de juego, ludoteca, juegoteca, que hizo el aguante, que hizo borde, aquel lugar que preservó, ¿tal vez fue digno de ser preservado?

Durante la búsqueda de material para la realización del trabajo, encontramos el relato de un mito griego: Las Moiras (2012) son tres hermanas: Cloto (la más joven, la que hila), Láquesis (la del medio, la que teje el destino) y Átropos (la más vieja, la que cor-

policía y un vecino, se desata una secuencia de hechos: se quema el destacamento policial y, luego, algunas personas entraron al Centro Comunitario, saquearon y destrozaron gran parte del lugar. Después, quemaron el jardín de la Escuela Primaria, intentaron sacar las computadoras de la escuela secundaria. Ese fin de semana hubo más muertes y enfrentamientos, una situación social estallada que los trabajadores de distintas instituciones veníamos denunciando, pero que nadie pareció estar dispuesto a escuchar. 
ta los hilos). Las tres deciden, tejen y entretejen el hilo de la vida. En el momento del nacimiento del niño, son la personificación del destino. Estas deidades deben asegurarse de que el destino se cumpla según lo planeado, incluso el destino de los dioses. Ellas hilan su destino y luego predicen su futuro.

Cuando leíamos este mito, recordábamos la fuerza y firmeza con la que llegan algunos diagnósticos de niños y niñas, algunos pronósticos formulados más en el orden de una sentencia certera y no sólo en términos médicos, sino en torno a la no posibilidad de construir un recorrido diferente al de sus padres, hermanos, familias.

La comprensión de una secuencia histórica exige la posibilidad de seriar los acontecimientos tanto como la de comparar los intervalos que separan los eventos entre sí. Esta operación requiere de un operación psíquica, la de secuenciar, que como vimos en los relatos y en los decires de los niños, son posibles en el momento en que comienzan a instalar algunas preguntas en torno al tiempo. Algo respecto de alguna fijeza, podríamos preguntar: ¿habría quitado en estos niños, la posibilidad de formularlas?

Algunos hilos de destino se comienzan a romper, para posibilitar anudar otros presentes, pasados y futuros. Este dispositivo grupal, oficiando de referencia (incluso para las familias ante tanta discontinuidad en los profesionales de las instituciones) y de pertenencia, respetuoso del rito construido, tal vez posibilitó pasar de la desafiliacióndestino precedido a lo grupal y así, en este movimiento pudo construirse un mientras tanto, que abrió a poder pensar otros modos de estar, otros modos de vivir, de jugar, de imaginar con otros.

\section{Bibliografía}

- BASILE, Marta (2012) Barriletes En Bandada En Salud Ele-Mental. Con Toda La Mar Detrás. Fernando Ulloa. Buenos Aires. Argentina. Zorzal

- MiTO DE LAS MOIRAS: Http:// www.Cuentos-Infantiles.Org/ElMito-De-Las-Moiras/ Fri, 28 Dec 2012 20:50:34 -0300

- MOFFATT, Alfredo: "Los Chicos De La Calle: El Psiquismo Acrónico”. Primera Parte. Psicopatología Y Sociopatología. Revista Psicología Social Hoy. Marzo-Abril 1991 Http://www.Moffatt.Com.Ar/

- RODULFO, Ricardo (1999) El Niño Y El Significante. Un Estudio Sobre Las Funciones Del Jugar En La Contitución Temprana. 5ta. Reimpresión. Paidós 